\title{
EBP Algorithm Can Work With Hard Limiters
}

\author{
Vojislav Kecman \\ Fulbright Visiting Scientist, MIT, Dept. of Mech. Eng. \\ Cambridge, MA 02139-4307, USA
}

The most important requirement for application of the error back-propagation (EBP) algorithm in multilayerd feed-forward artificial neural network (ANN) is that the neuron's activation function is a nonlinear, smooth and (sometime even nondecreasing) differentiable function. This is not valid anymore. This paper shows why and how simple treshold logic unit (TLU), or signum function, can be used instead of sigmoidal (or other different differentiable) activation function. The introduction of TLU is based on the understanding that the hidden layer is feeding the output layer with linearly separable patterns from its image space. To better understand the motivation for using TLU in multilayerd ANN and what is, actually, the hidden layer doing while facing nonseparable patterns of data, we use the famous XOR problem which, being two dimensional, can be explained in a graphical way, [3]. Thus, it is very important to realize that the output layer deals with linearly separable patterns, or that this layer is not separating data from input space but rather from internal, "invisible" representation or from, so called, image space and patterns in this space are separable.

Application of TLU in output layer to the XOR problem resulted in very fast convergence and without any error in just 12 leaming cycles. Taking TLU for output layer nodes, equation for calculation of output deltas should be changed and standard algorithm for discrete perceptron was used without need for the value of the derivative of the activation function anymore. It is worthwhile to mention that the rest of the EBP algorithm remains the same. But there was still the unanswered question that could EBP algorithm have been more effective and maybe faster had the TLU been used in hidden layer, too. The answer was positive, but the solution in this case is now a little more complicated and diverges from theoreticaly 'clean' framework (we had in the previous first change of the EBP algorithm) to more ineuristics. With that full-discrete ANN we solved XOR problem in just 5 cycles.

The paper has shown how TLU can be succesfully applied in ANN's at both output as well as the hidden layer. EBP algorithm is still applicable without global changes but the expressions for calculation of delta values should be accordingly changed. Significant reduction in number of iterative learning cycles is achieved making the algorithm faster and without any error. More practical investigation (primarely on larger scale problems) should be done in order to test robustness and applicability of proposed changes.

\section{REFERENCES}

[1] D. E. Rumelhart, J. L. McClelland, PDP, Vol. 1, MTT Press, 1986

[2] P. J. Werbos, Beyond regresion, Ph.D. dissertation, Harvard University, 1974

[3] J.M. Zurada, Introduction to Artificial Neural Systems, West Pub.Comp.,St. Paul,1992

[4] T. Poggio, F. Girosi, Networks for Approximation and Learning, Proc. of the IEEE, Sept., 1990 